\title{
ASTEP: Towards the detection and characterization of exoplanets from Dome C
}

\author{
N. Crouzet ${ }^{1}$, T. Guillot ${ }^{1}$, K. Agabi ${ }^{2}$, J.-B. Daban ${ }^{2}$, L. Abe ${ }^{2}$, D. Mekarnia ${ }^{2}$, \\ J.-P. Rivet ${ }^{1}$, Y. Fantë̈-Caujolle ${ }^{2}$, F. Fressin ${ }^{3}$, C. Gouvret ${ }^{2}$, F.-X. Schmider ${ }^{2}$, \\ F. Valbousquet ${ }^{4}$, A. Blazit ${ }^{2}$, H. Rauer ${ }^{5,6}$, A. Erikson ${ }^{5}$, T. Fruth ${ }^{5}$, \\ S. Aigrain ${ }^{7}$, F. Pont $^{8}$, M. Barbieri ${ }^{9}$ \\ ${ }^{1}$ Université Nice Sophia Antipolis, CNRS, Observatoire Côte d'Azur, France \\ [crouzet@oca.eu] \\ ${ }^{2}$ Université Nice Sophia Antipolis, CNRS, Observatoire Côte d'Azur, France \\ ${ }^{3}$ Harvard-Smithsonian Center for Astrophysics, Cambridge, MA, US \\ ${ }^{4}$ Optique et Vision, Juan les Pins, France \\ ${ }^{5}$ DLR Institute for Planetary Research, Berlin, Germany \\ ${ }^{6}$ Center for Astronomy and Astrophysics, TU Berlin, Germany \\ 7 Oxford Astrophysics, Department of Physics, UK \\ 8 School of Physics, University of Exeter, UK \\ ${ }^{9}$ Universitá di Padova, Dipartimento di Astronomia, Italy
}

\begin{abstract}
.
The ASTEP project (Antarctic Search for Transiting ExoPlanets), aims at testing the quality of the Dome $\mathrm{C}$ site in Antarctica for photometry in the visible, as well as detecting and characterizing transiting exoplanets. A dedicated telescope, ASTEP400, has been developped and installed at Concordia. The first campaign took place during the winter 2010, and the telescope functionned nominally during all the winter. A first analysis of the data leads to a precision of 189 and $205 \mathrm{ppm}$ for WASP-19 and WASP-18 respectively, for continuous observations during 1 month. This shows that extremely high precision photometry is achievable from Dome C.
\end{abstract}

\section{Introduction}

The Concordia base in Dome C, Antarctica, is an extremely promising site for photometry due to a 3-month long night during the Antarctic winter and favorable atmospheric conditions. The ASTEP project aims at testing the quality of the Dome $\mathrm{C}$ site for photometry in the visible, as well as detecting and characterizing transiting exoplanets (Fressin et al. 2005). A first instrument, ASTEP South, has been observing during the 2008, 2009 and 2010 winters (Crouzet et al. 2010). The heart of the project is ASTEP400, a telescope entirely designed and built to perform high-precision photometry under the extreme conditions of the Antarctic winter. The first campaign with ASTEP400 started in March 2010. In the first section we give an overview of the ASTEP400 instrument. Then, we use preliminary lightcurves to evaluate the photometric quality of Dome C. 


\section{An instrument for Antarctica}

ASTEP400 is a $40 \mathrm{~cm}$ Newton telescope with a $1^{\circ} \times 1^{\circ}$ field of view. The structure is a Serrurier made with carbon-epoxy tubes, with Invar sleeves at each end. This combination of materials guarantees a very low coefficient of thermal expansion for the distance between the primary and the secondary mirror. Our calculations show that, with a temperature increase of $30 \mathrm{~K}$, this distance increases by less than $20 \mu \mathrm{m}$. The primary and secondary mirrors are in Zerodur. A thermalized box is fixed on this structure. It contains mainly a science and a guiding camera, a dichroic beam-splitter to separate both channels, and a field corrector on the science channel. This box is closed by a double-glass window and heated at $-20^{\circ} \mathrm{C}$. The structure and the camera box are shown figure 1. The science camera is a Proline from FingerLake Instrumentation, equipped with a Kodak KAF-16801E front-illuminated $4096 \times 4096$ pixels CCD; see Crouzet et al. (2007) for the choice of the camera. Its quantum efficiency peaks at $67 \%$ at $670 \mathrm{~nm}$, with a mean of $50 \%$ in the spectral range $600-800 \mathrm{~nm}$. The pixel size is $9 \mu \mathrm{m}$. The total instrumental bandpass is equivalent to a large $\mathrm{R}$ band (600-900 $\mathrm{nm}$ ). The PSF is designed to be defocused, with a FWHM (Full Width Half Maximum) between 3 and 4 pixels. The mount is a 3600GTO equatorial mount from Astro-Physics, modified to operate down to $-80^{\circ} \mathrm{C}$. For example, all the grease has been changed. The instrument is inside a dome. The instrument is described in more details in Daban et al. (2010).

ASTEP400 has been installed at the Concordia station in December 2009, at the ground level (figure 2). The first campaign started in March 2010. The telescope functionned nominally during all the winter (until the end of September), and the data are of good quality. Due to a limited internet connection with the Concordia base, the data are stored on site until the end of the winter. The analysis presented here is thus done without a first look to the data. The reduction routines are written in Nice mainly by Djamel Mekarnia, and sent to Karim Agabi who is spending the winterover at Concordia. Photometry is then performed on the data in a blind way, and the obtained lightcurves are sent back to Nice. At most, we can get $\sim 2000$ lightcurves each day.

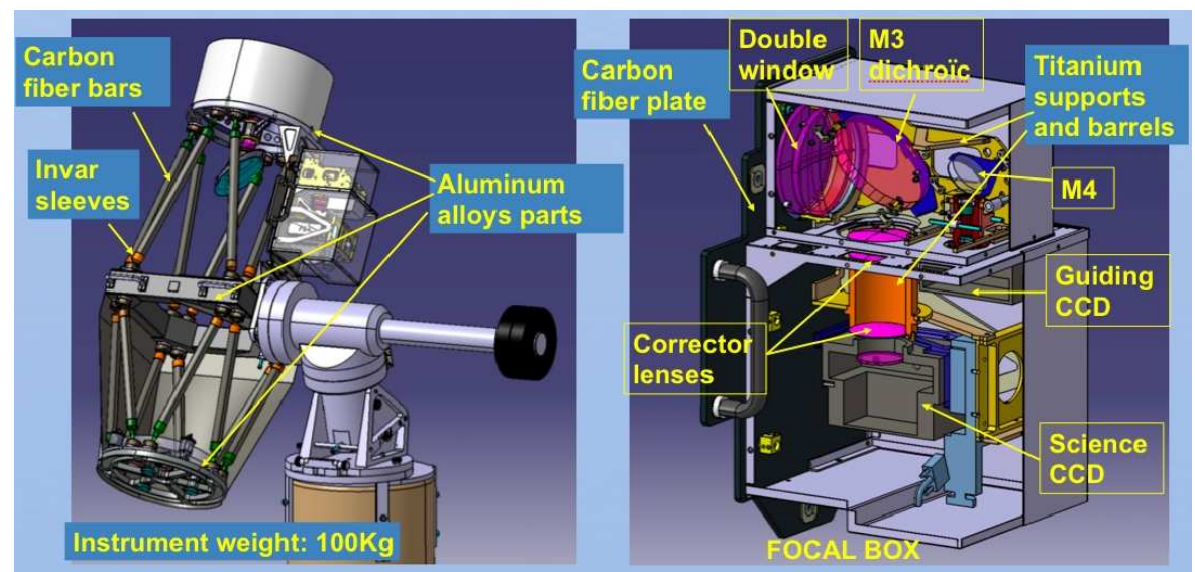

Figure 1: General view of the mechanical structure (left) and the camera box (right) of ASTEP 400 . 


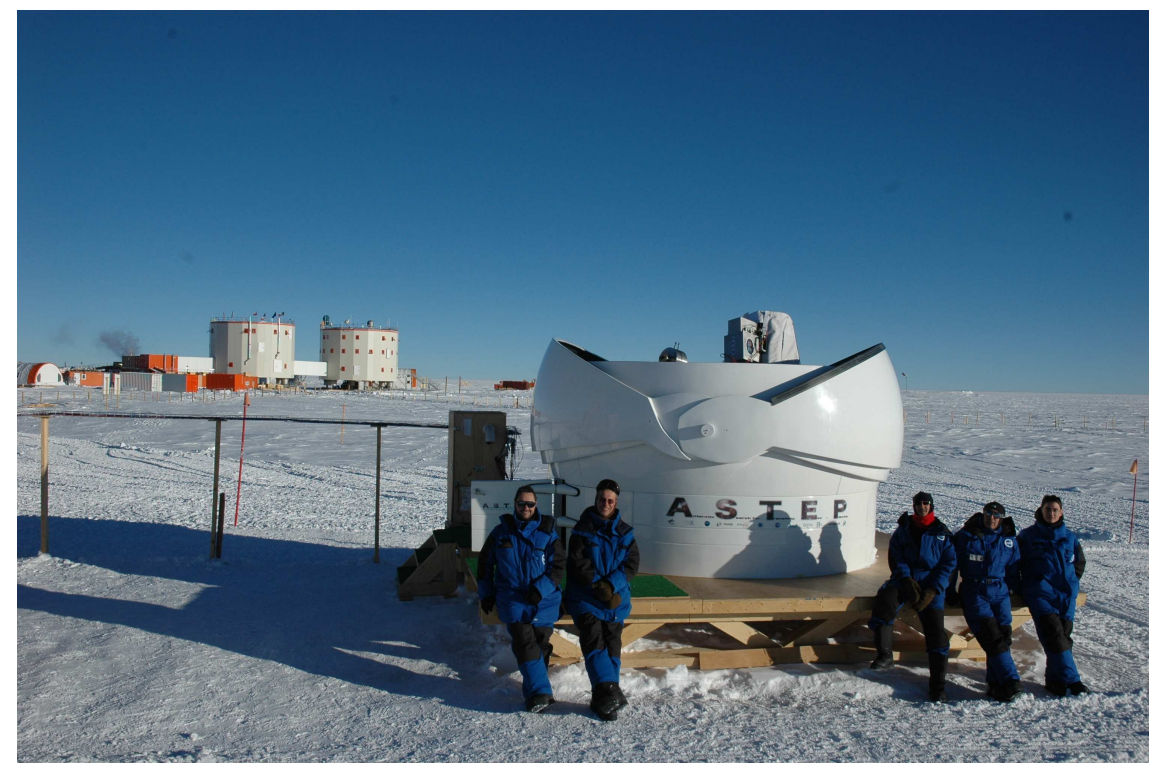

Figure 2: Installation of ASTEP400 at Concordia in December 2009.

\section{A first analysis of the photometric quality at Dome C}

The campaign is divided into different kinds of observations. For a first evaluation of the quality of Dome C for photometry, we present here i) observations of stellar dense fields, and ii) continuous observations of specific targets.

\subsection{Observations of stellar dense fields}

The observations of well chosen stellar dense fields is a way to detect transiting exoplanets. Figure 3 shows a typical RMS-magnitude diagram obtained from 1 day data with ASTEP400. The field contains $\sim 20000$ stars up to magnitude 16, only $\sim 2000$ are shown here. The exposure time is $70 \mathrm{~s}$, no binning is applied. We reach a precision of $2 \mathrm{mmag}$ for stars of magnitude 12 over 1 day. Moreover, a complete analysis of the data should improve this precision. We therefore show that high precision photometry is feasible from Dome C.

\subsection{Continuous observations of specific targets}

Observations of known transiting planets have been made to test the photometry and try to detect the secondary transits and the phase curve of the planet. We chose 2 target stars: WASP-19 and WASP-18.

\section{WASP-19}

WASP-19 is a solar type star of magnitude 12.3 harboring a hot-Jupiter transiting planet of period $1.789 \mathrm{~d}$. This target is observed continuously from April 30 to May 23, 2010. The exposure time is $130 \mathrm{~s}$. Flat fields are taken on the dark or twilight sky with an automatic procedure, and stars are eliminated by a median filter. Photometry is performed on a day-by-day basis, by summing bright pixels. A polynomial function of 


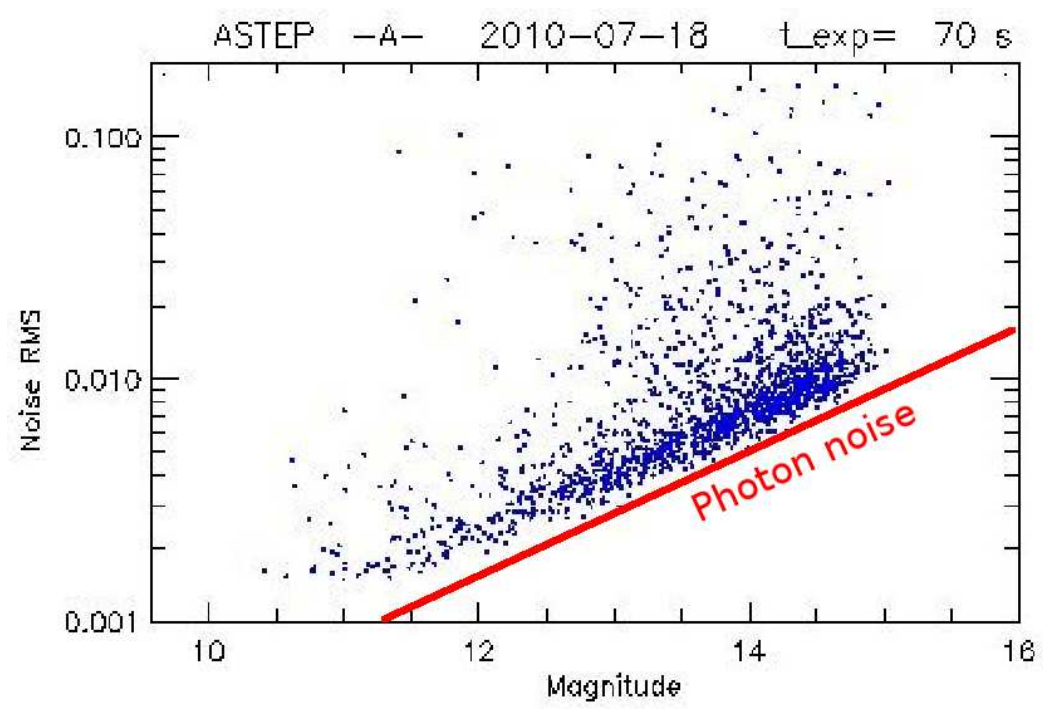

Figure 3: Typical RMS-magnitude diagram obtained from 1 day data with ASTEP400, for 70 s exposures. Only are shown stars up to magnitude $\sim 15$, since all lightcurves can not be sent to Nice.

degree between 2 and 4 is fitted and substracted to the 1-day lightcurves, to eliminate low frequency variations (this correction is applied to search for the secondary transit, but will not be applied to search for the phase curve).

Figure 4 shows the lightcurve of WASP-19 over the 24 days of observations. The typical dispersion is $1.4 \mathrm{mmag}$ over 1 day. Individual transits of $2 \%$ depth appear clearly. As a comparison, observations of WASP-19 with the Faulkes Telescope South (2 m diameter, Siding Spring Observatory, Australia) reach a precision of $1.3 \mathrm{mmag}$ in the $\mathrm{z}$ band (Hebb et al. 2010). Both precisions are comparable. Our $40 \mathrm{~cm}$ telescope located at Dome $\mathrm{C}$ is thus competitive with a $2 \mathrm{~m}$ telescope located in one of the best observatories in the world. This shows the very high quality of Dome $\mathrm{C}$ for photometry.

To infer further the photometric precision that can be reached from Dome $\mathrm{C}$, we fold the curve in phase, to take advantage of the high phase coverage. The lightcurve is smoothed over $1.3 \mathrm{~h}$ (the transit duration), and the dispersion is computed using a MAD (Mean Absolute Deviation). This results in a precision of $189 \mathrm{ppm}$ at $1 \sigma$, which is exceptional for photometric observations in the visible from the ground. At this stage, we can already put an upper limit on the brightness temperature of WASP$19 \mathrm{~b}$ in the visible: $\Delta T_{b, v i s}<1.24 T_{e q}$, where $T_{e q}$ is the equilibrium temperature of the planet. The analysis is under improvement to identify the secondary transit. In any case, this precision shows that the extremely high precision needed for this kind of observations can be reached at Dome C.

WASP-18

WASP-18 is a solar type star of magnitude 9.3 harboring a 10.4 Jupiter masses transiting planet of period $1.789 \mathrm{~d}$. This target is observed continuously from June 16 to July 9, 2010. The exposure time is $15 \mathrm{~s}$. The analysis is done in the same way as for WASP-19. 

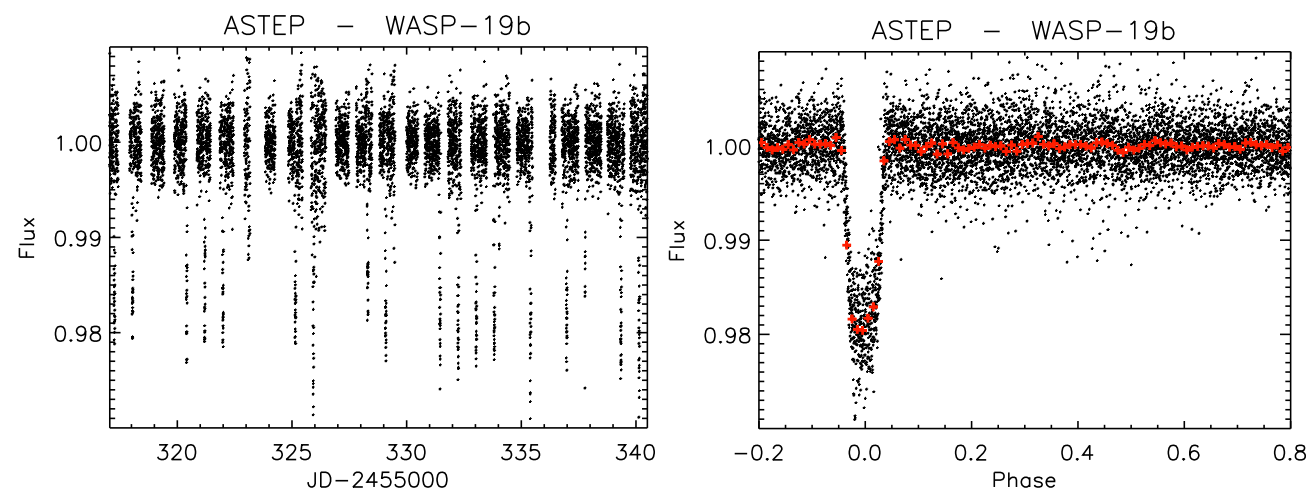

Figure 4: Lightcurve of WASP-19 obtained with ASTEP400 between April 30 and May 23, 2010 (left). The curve is folded in phase (right), and a mean is computed for each interval of $1 / 100^{\text {th }}$ of the orbital period, i.e. $11 \mathrm{mn}$ (red).

Figure 5 shows the lightcurve of WASP-18 over the 23 days of observations. The typical dispersion is 0.8 mmag over 1 day. Individual transits of $0.88 \%$ depth appear clearly. As a comparison, observations of WASP-19 with the Danish Telescope $(1.5 \mathrm{~m}$ diameter, La Silla Observatory, ESO, Chile), in the V band with 80 s exposure time, reach a precision of 0.47 to $0.83 \mathrm{mmag}$ (Southworth et al. 2009). Again, our $40 \mathrm{~cm}$ telescope at Dome $\mathrm{C}$ is competitive with larger telescopes in the best observatories.

The lightcurve is phased, smoothed over $1.6 \mathrm{~h}$ (the transit duration), and the dispersion is computed using a MAD. The precision is $205 \mathrm{ppm}$ at $1 \sigma$. This precision is close to WASP-19 whereas the star is 3 magnitude brighter. We explain that by less favorable atmospheric conditions during the observations and a lack of bright comparison stars in the field of view. This precision is still exceptional from the ground. We can also put an upper limit on the brightness temperature of WASP-18 b in the visible: $\Delta T_{b, v i s}<1.26 T_{e q}$, where $T_{e q}$ is the equilibrium temperature of the planet.
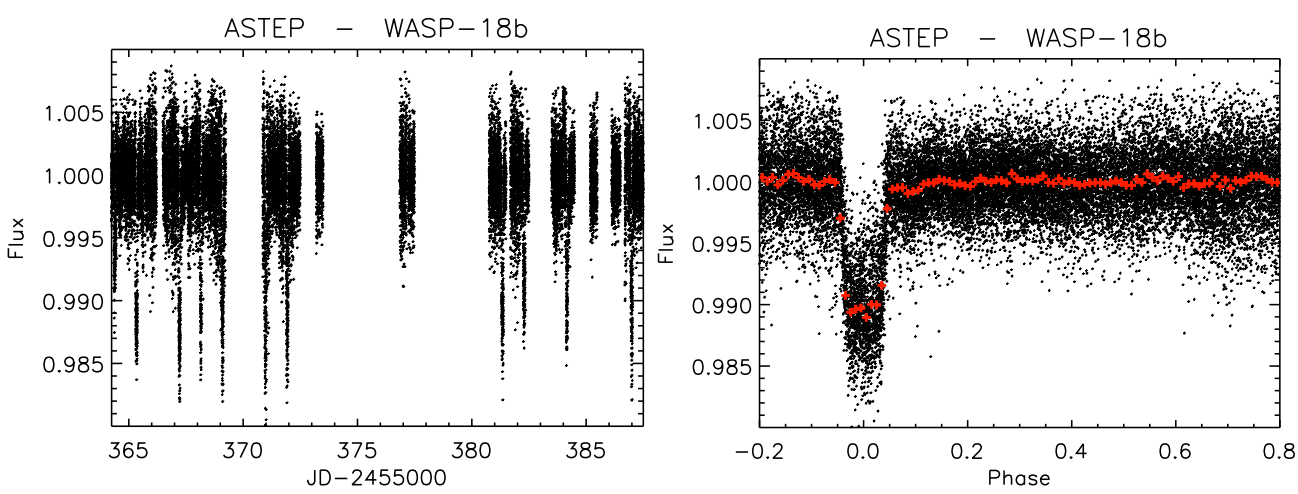

Figure 5: Lightcurve of WASP-18 obtained with ASTEP400 between June 16 and July 9, 2010 (left). The curve is folded in phase (right), and a mean is computed for each interval of $1 / 100^{\text {th }}$ of the orbital period, i.e. $14 \mathrm{mn}$ (red). 


\section{EPJ Web of Conferences}

\section{Conclusion}

We showed that a dedicated telescope such as ASTEP400 can operate nominally during all the winter at Dome $\mathrm{C}$, and perform photometry of very high quality. In spite of a very low internet connection that prevents us from getting the data, an on-site analysis leads to the first lightcurves. First estimates of the photometric precision that can be reached with ASTEP400 are very encouraging, in particular by observing continuously specific targets such as WASP-19 and WASP-18. The precision obtained on the curves in phase is extremely high for an instrument observing from the ground in the visible. This shows that very high precision photometry is achievable from Dome C. This analysis is under improvement to search for the secondary transit and the phase curve of the planets.

Acknowledgements. The ASTEP project is funded by the Agence Nationale de la Recherche (ANR), the Institut National des Sciences de l'Univers (INSU), the Programme National de Planétologie (PNP), and the Plan Pluri-Formation OPERA between the Observatoire de la Côte d'Azur and the Université de Nice-Sophia Antipolis. The entire logistics at Concordia is handled by the French Institut Paul-Emile Victor (IPEV) and the Italian Programma Nazionale di Ricerche in Antartide (PNRA). The research grant for N. Crouzet has been supplied by the Région Provence Alpes Côte d'Azur, the Observatoire de la Côte d'Azur, and the Centre National de la Recherche Scientifique (CNRS). See http://fizeau.unice.fr/astep/ for more information about the ASTEP project.

\section{References}

Crouzet, N., Guillot, T., Agabi, A., Rivet, J., Bondoux, E., et al., 2010, A\&A, 511, 36

Crouzet, N., Guillot, T., Fressin, F., Blazit, A., \& the ASTEP team. 2007, Astronomische Nachrichten, 328, 805

Daban, J., Gouvret, C., Guillot, T., Agabi, A., Crouzet, N., et al., 2010, in Society of PhotoOptical Instrumentation Engineers (SPIE) Conference Series, Vol. 7733, Society of Photo-Optical Instrumentation Engineers (SPIE) Conference Series

Fressin, F., Guillot, T., Bouchy, F., Erikson, A., Gay, J., et al., 2005, in EAS Publications Series, Vol. 14, EAS Publications Series, ed. M. Giard, F. Casoli, \& F. Paletou, 309312

Hebb, L., Collier-Cameron, A., Triaud, A. H. M. J., Lister, T. A., Smalley, B., et al., 2010, ApJ, 708, 224

Southworth, J., Hinse, T. C., Dominik, M., Glitrup, M., Jorgensen, U. G., et al., 2009, ApJ, 707,167 